Rev. salud pública. 14 sup (1): 1-17, 2012

Artículos Originales/Original Articles

\title{
Caracterización socio-laboral y de aseguramiento en salud de dos grupos de trabajadores cesantes de Medellín- Colombia 2004 y 2007
}

\section{Characterization socio-occupational and health insurance of two groups of unemployed workers from Medellin-Colombia 2004 and 2007}

Álvaro Cardona, Emmanuel Nieto L y Luz M. Mejía O.

Facultad Nacional de Salud Pública. Universidad de Antioquia. Medellín, Colombia. alvarocardona66@

hotmail.com, emmanuel@saludpublica.udea.edu.co, luzmmejia@saludpublica.udea.edu.co

Recibido 31 Agosto 2009/Enviado para Modificación 3 de Mayo 2010/Aceptado 11 Mayo 2010

\section{RESUMEN}

Objetivo Analizar las condiciones socio-laborales y de aseguramiento en salud de dos grupos de trabajadores que perdieron su empleo en la ciudad de MedellínColombia en dos periodos (2004 y 2007), a fin de contribuir al diseño de políticas públicas alternativas que afronten eficazmente el problema de desprotección en salud del trabajador cesante y su grupo familiar.

Metodología A partir de la información primaria, recolectada mediante el mismo instrumento aplicado a dos muestras independientes de trabajadores que perdieron su empleo en la ciudad de Medellín en 2004 ( $n=267)$ y en 2007 ( $n=198)$, se realizó un estudio descriptivo de corte transversal utilizando técnicas de investigación cuantitativa de análisis univariado y bivariado.

Resultados Tanto en 2004 como en 2007 la eventualidad de que los trabajadores cesantes quedaran sin aseguramiento en salud estuvo asociada en buena medida a variables de sexo, edad, educación, estrato socioeconómico, posición ocupacional, nivel salarial y duración del desempleo. Su disposición de acceder a recursos del microcrédito o a un micro seguro, propuestos a la Administración Municipal como políticas públicas para mantener su afiliación como cotizantes en el régimen contributivo en salud, fue altamente favorable en ambos grupos.

Conclusiones El perfil socio-laboral de los trabajadores cesantes guarda correspondencia en ambos grupos, aunque se presentan diferencias relevantes en las condiciones de aseguramiento en salud. Se concluye que la vulnerabilidad de los trabajadores cesantes que quedan excluidos del aseguramiento en salud demanda de la sociedad y del Estado políticas públicas alternativas para su protección. 
Palabras Clave: Desempleo, seguro de salud, seguridad social (fuente: DeCS, BIREME)

\section{ABSTRACT}

Objective Analyzing the socio-occupational and health insurance for two groups of workers who lost their job in the city of Medellin, Colombia in two periods (2004 and 2007), in order to help design alternative public policies that effectively confront the problem of vulnerability in health of unemployed workers and their families. Methodology Based on primary information, collected through the same instrument applied to two independent samples of workers who lost their job in the city of Medellin in $2004(n=267)$ and $2007(n=198)$, a descriptive cross-sectional study was made using quantitative research techniques of univariate and bivariate analysis.

Results In both 2004 and 2007 studies the possibility that unemployed workers remain without health insurance was largely associated to variables of sex, age, education, socioeconomic status, occupational status, salary level and duration of unemployment. Their willingness to access to micro credit resources or a micro insurance, proposed to the municipal government as public policies to maintain their membership as contributors to the contributory regime, was highly favorable in both groups.

Conclusions The socio-occupational profile of unemployed workers keeps correspondence in both groups, although there are significant differences in the conditions of health insurance. We conclude that the vulnerability of unemployed workers excluded from the health insurance demands of society and State alternative public policies for their protection.

Key Words: Unemployment, insurance, social security (source: MeSH, NLM)

E l derecho a la protección en salud de los ciudadanos ha sido históricamente una de las preocupaciones de la seguridad social y de la salud pública internacional. Este debate abarca las políticas de bienestar que deben desarrollar los gobiernos y la sociedad para garantizar el acceso a los servicios de salud con calidad y oportunidad (1).

No obstante las reformas a los sistemas de salud de América Latina y el Caribe en las dos últimas décadas, un porcentaje importante de la población se encuentran excluida de mecanismos de protección social (2). En tal sentido, se ha propuesto para estos países el concepto de la Extensión de la Protección Social en Salud como la garantía que la sociedad otorga, por conducto de los poderes públicos, para que los individuos puedan satisfacer sus necesidades y demandas de salud, mediante el adecuado acceso a los servicios, sin que la capacidad de pago sea un factor restrictivo (3). 
Este concepto incluye tres condiciones esenciales para garantizar la protección social en salud: a. Acceso a los servicios, es decir, que exista la oferta necesaria para la provisión de servicios de salud y que las personas puedan tener acceso física y económicamente a ellos; b. Seguridad financiera del hogar, lo cual sugiere que el financiamiento de los servicios de salud no constituya una amenaza para la estabilidad económica de las familias ni para el desarrollo de sus miembros; c. Dignidad en la atención en salud para que la misma se provea con calidad y en condiciones de respeto a las características raciales, culturales y económicas de los usuarios, definidas mediante un proceso de diálogo social (3). Estas tres dimensiones de la protección social son imprescindibles y la ausencia de una o más de ellas no sólo determina alguna forma de exclusión en salud, sino que pone a las familias en alto riesgo de pérdida de su patrimonio y por tanto de caer en la pobreza o aumentarla.

En Colombia las dificultades para alcanzar la universalización del aseguramiento en salud están estrechamente asociadas a las restricciones que se originan en una precaria dinámica del mercado de trabajo (4). Pese a que para 2004 y 2007 el país retoma la senda de un mayor crecimiento económico, con tasas de 5,3 \% y 6,9 \% en ambos años, se mantienen altos los índices de desempleo, pobreza y desigualdad.

Aún en ciudades con una fuerte vocación industrial como Medellín, la recuperación de la actividad productiva no logró revertir dicho deterioro socio-laboral. En estos dos años, la ciudad registró en su orden tasas de desempleo de 15,4 \% y 12,3 \%. Las tasas de empleo informal, por su parte, alcanzaron respectivamente niveles del 55,2 \% y 51,5 \% (5).

La falta de oportunidades laborales es uno de los factores que más está incidiendo en los niveles de pobreza y desigualdad que se registran en Medellín. Para el 2007, según mediciones de los ingresos, el 38,5 \% de la población de esta ciudad se mantuvo en condiciones de pobreza. Sin embargo, para ese mismo año las autoridades locales estiman que el 42,3\% de la población clasifica en los niveles 1 y 2 del Sistema de Identificación de Potenciales Beneficiarios de Programas Sociales SISBEN. El Coeficiente de Gini, que mide la desigualdad en la distribución del ingreso, registró para ese año un nivel de 0,52 , tanto o más alto que lo observado en el nivel nacional (6). 
En este contexto emergen condiciones sociales y económicas de los trabajadores cesantes (TC) y sus familias que no sólo se convierten en barreras de acceso a los servicios de salud, sino que les aumenta la probabilidad de deslizarse a mayores niveles de pobreza (7-9). Resulta obvio que si los trabajadores pierden su empleo y no disponen de aseguramiento en salud al momento de sufrir ellos o algún miembro de su grupo familiar alguna enfermedad que implique altos costos para su atención, tendrán que hacer esfuerzos económicos soportados en su escaso patrimonio familiar, poniéndose en riesgo de empobrecimiento. Esto sin contar que en caso de no disponer de esos recursos patrimoniales, tendrán que soportar la enfermedad y el deterioro de su bienestar, arriesgando incluso su vida.

En el marco de la seguridad social colombiano, diversos estudios muestran que el problema de cobertura del sistema obedece a razones estructurales y de desempeño, siendo la principal de ellas la carencia de estrategias y políticas para el aseguramiento de la población de estratos medios no vinculada al empleo formal. Adicionalmente, no se ha contemplado la real dimensión de empleo y desempleo del país, en tanto que el diseño del sistema de salud supone que todos los trabajadores independientes e informales tienen capacidad económica de cotizar al sistema (10-13).

Entre los estudios del desempleo en Colombia, lo predominante en su análisis ha sido la relación con el comportamiento macroeconómico y los factores sociodemográficos que afectan la participación en el mercado laboral. Además de mostrar las tendencias del desempleo y los grupos más vulnerables, algunos indagan sobre sus nexos con los desequilibrios del sistema educativo, los programas de recalificación profesional y los mecanismos de intermediación laboral $(14,15)$. Otros, han avanzado en el análisis del impacto que sobre esta variable han generado los procesos de reformas estructurales y los cambios en las normas laborales, específicamente se identifican las limitaciones de oferta de nuevas fuentes de empleo por los altos costos de las contribuciones parafiscales $(16,17)$.

Algunos de esos estudios explican la relación entre el desempleo y el aseguramiento en salud, en forma descriptiva de los niveles del aseguramiento en los distintos regímenes de salud y las condiciones sociolaborales de los trabajadores. Se sostiene que la forma de financiación del aseguramiento fundamentado en la cotización sobre la nómina, supone 
una gran vulnerabilidad del sistema en relación con el comportamiento de la actividad productiva y la evolución de la masa de asalariados y desempleados (18-22).

Algunos analistas resaltan los problemas de diseño del Sistema General de Seguridad Social en Salud (SGSSS) colombiano, argumentando que aún bajo condiciones excepcionales de crecimiento económico, las coberturas tampoco lograrían avanzar sustancialmente, en tanto buena parte de los recursos se queda en manos de los intermediarios (23-25).

Nuestra investigación se ha soportado en la hipótesis de que gran parte de los TC, especialmente aquellos de los estratos socioeconómicos de clase media, quedan excluidos del aseguramiento en salud, porque no disponen de recursos suficientes para mantener su continuidad en el régimen contributivo, ni hacen parte de la población más pobre que se prioriza para afiliarse en el régimen subsidiado.

Lo anterior, ha motivado el desarrollo de una línea de investigación que intenta comprender la relación entre las condiciones del entorno socioeconómico de los TC y sus condiciones de protección social en salud después de perder su empleo. Específicamente hemos avanzado en la exploración de las condiciones socio-laborales y de aseguramiento en salud de dos grupos de TC de la ciudad de Medellín, en 2004 y 2007, a fin de proponer alternativas de política pública para la protección social en salud a este grupo poblacional.

\section{MÉTODOS}

En 2004 y 2007 se realizaron estudios descriptivos de corte transversal haciendo uso de técnicas de investigación cuantitativa, sobre la base de información primaria recolectada a partir de la aplicación de un mismo instrumento a dos muestras independientes de la población de TC de la ciudad de Medellín. Las muestras aleatorias simples que dieron soporte a los dos estudios se estimaron en 267 trabajadores en 2004 y 198 trabajadores en 2007. En ambos casos la muestra fue seleccionada a partir de las bases de datos del Servicio Público de Empleo del Servicio Nacional de Aprendizaje (SENA) y de los registros de desafiliación por pérdida de empleo de las cuatro Entidades Promotoras de Salud (EPS) más grandes que operan en la ciudad de Medellín. 
Las dos muestras, aunque independientes, permiten la comparación en tanto son representativas de la población de TC reportada en las bases de datos de las instituciones ya señaladas, a las cuales se les aplicó la misma encuesta que permitió levantar información sobre sexo, edad, educación, estrato socioeconómico, empleo-desempleo, tiempo de desempleo, actividad económica, tamaño de la empresa, posición ocupacional, salario, duración del empleo y tipo de contrato del último empleo. También se indagó sobre la afiliación a la seguridad social en salud, según regímenes de afiliación y según la condición de cotizante o beneficiario.

En ambos estudios, el criterio de inclusión era que los trabajadores hubieran perdido su empleo en el respectivo periodo. Se consideró trabajador cesante TC, de acuerdo con el Departamento Nacional de Estadística DANE (26), a la persona que habiendo trabajado por lo menos durante dos semanas consecutivas se encontraba desocupado pero buscando empleo.

Se consideró asegurado en salud al TC que estuviera afiliado a alguno de los regímenes del SGSSS, de acuerdo con la Ley 100 de 1993 que establece el régimen contributivo para la población trabajadora y/o con capacidad para cotizar y el régimen subsidiado para la población pobre sin capacidad de contribuir al Sistema. Adicionalmente, incorpora unos regímenes especiales o de excepción para las Fuerzas Militares y Policía, los trabajadores de la Empresa Colombiana de Petróleo ECOPETROL y el Magisterio.

El análisis de la información se hizo de la siguiente manera: Análisis Univariado que permitiera identificar el perfil sociolaboral y de aseguramiento en salud de los TC, utilizando medidas de tendencia central y de dispersión para las variables cuantitativas; para las variables nominales se utilizaron frecuencias absolutas y frecuencias relativas. Análisis Bivariado para establecer las diferencias estadísticamente significativas entre las proporciones de afiliación o no al sistema de salud según el perfil sociolaboral. Con ese propósito se aplicó la prueba Chi cuadrado de bondad de ajuste para cada una de las variables nominales. En todos los casos se trabajó con un nivel de significación estadística del $95 \%$.

La base de datos y las diferentes pruebas de significación estadística se gestionaron en el software SPSS versión 19.0. 


\section{RESULTADOS}

Características socio-laborales de los trabajadores cesantes de la ciudad de Medellín, 2004 y 2007

En la tabla 1 semuestran las estadísticas que permiten una amplia caracterización sociolaboral de los TC en los dos años analizados. Se destacan las similitudes del perfil de estos trabajadores en ambos períodos en lo que respecta a edad, actividad económica, tamaño de empresa y posición ocupacional.

En ambos periodos, se trataba de una población trabajadora cesante relativamente joven, con un promedio de edad de 32 años y con algo más de la mitad con edades inferiores a los 30 años. Aunque entre estos trabajadores predominaba la actividad de servicios, cerca del $20 \%$ de ellos tuvieron experiencia laboral en el sector de la industria, fundamentalmente en el sector formal de la economía, es decir en empresas con tamaño superior a los 10 trabajadores. Su procedencia del sector formal de la economía se subraya con la fuerte proporción que declaró haber quedado cesante de una labor que desempeñaba como empleado del sector privado.

Entre los dos grupos se presenta cierta disparidad en su distribución por sexo, educación, estrato socioeconómico, niveles salariales y duración del empleo-desempleo. En 2004 los TC se distribuyen por igual entre hombres y mujeres, pero para el 2007 se evidencia una mayor presencia de estas últimas. En el primer grupo predomina la formación técnica y tecnológica, seguida de la

formación primaria. En el segundo grupo tiene mayor presencia la formación secundaria seguida de la formación técnica y tecnológica; la formación en educación superior es sensiblemente mayor en el grupo de 2007.

En cuanto a la distribución por estrato, no obstante que en los dos años se muestra una mayor participación del nivel socioeconómico bajo, en 2004 la participación de este estrato es mucho más acentuada. Lo anterior se corresponde con el hecho de que la proporción de quienes devengaban los niveles salariales más bajos es mayor en 2004 que en 2007; en este último grupo cerca del $63 \%$ declaró haber recibido en su último empleo más de un salario mínimo legal. Los promedios salariales, que en ambos períodos representaban cerca de 1,3 veces el salario mínimo legal del respectivo año, fueron en su orden de \$ 473.508 y \$ 563.609 mensuales. 
Tabla 1. Caracterización socio-laboral de los TC de Medellín, 2004 y 2007

\begin{tabular}{|c|c|c|c|c|}
\hline \multirow{2}{*}{ Variables } & \multicolumn{2}{|l|}{2004} & \multicolumn{2}{|c|}{2007} \\
\hline & $\mathrm{n}=267$ & $\%$ & $\mathrm{n}=198$ & $\%$ \\
\hline \multicolumn{5}{|l|}{ Sexo } \\
\hline Mujer & 135 & 50,6 & 129 & 65,2 \\
\hline Hombre & 132 & 49,4 & 69 & 34,8 \\
\hline Edad & $(X=31,7$ años $)$ & & & (X=32 años) \\
\hline 29 o menos años & 138 & 51,7 & 108 & 54,5 \\
\hline 30 a 39 años & 68 & 25,5 & 44 & 22,2 \\
\hline 40 o más años & 61 & 22,8 & 46 & 23,2 \\
\hline \multicolumn{5}{|l|}{ Educación } \\
\hline Primaria & 62 & 23,7 & 34 & 17,2 \\
\hline Secundaria & 36 & 13,5 & 95 & 48 \\
\hline Técnico-tecnológico & 143 & 53,5 & 44 & 22,2 \\
\hline Superior & 26 & 9,7 & 25 & 12,6 \\
\hline \multicolumn{5}{|l|}{ Estrato socioeconómico } \\
\hline Bajo & 168 & 63 & 107 & 54 \\
\hline Medio-alto & 99 & 37 & 91 & 46 \\
\hline \multicolumn{5}{|l|}{ Sector económico } \\
\hline Industria & 53 & 19,9 & 39 & 19,7 \\
\hline Comercio & 72 & 27 & 61 & 30,8 \\
\hline Resto & 142 & 53,1 & 98 & 49,5 \\
\hline \multicolumn{5}{|l|}{ Tamaño de empresa } \\
\hline 1 a 10 trabajadores & 86 & 32,2 & 63 & 31,8 \\
\hline Más de 10 trabajadores & 182 & 67,8 & 135 & 68,2 \\
\hline \multicolumn{5}{|l|}{ Posición ocupacional } \\
\hline Empleado s-privado & 232 & 86,9 & 168 & 84,4 \\
\hline Empleado s-público & 22 & 8,2 & 14 & 7,1 \\
\hline Resto $\left(^{*}\right)$ & 13 & 4,9 & 16 & 8,1 \\
\hline Salario último empleo $\left.{ }^{* *}\right)$ & $(X=\$ 473.508)$ & & & $(X=\$ 563.609)$ \\
\hline 1 smlv o menos & 139 & 52,3 & 74 & 37,4 \\
\hline Entre 1 y $1,5 \mathrm{SMLV}$ & 79 & 29,7 & 71 & 35,9 \\
\hline Más de 1,5 SMLV & 48 & 18 & 53 & 26,8 \\
\hline Duración del desempleo & $(X=8,1)$ & & $(X=10,3)$ & \\
\hline Menos de 6 meses & 128 & 48 & 61 & 30,8 \\
\hline Entre 6 y 12 meses & 63 & 23,6 & 47 & 23,7 \\
\hline 12 meses y más & 76 & 28,4 & 90 & 45,5 \\
\hline
\end{tabular}

Estos niveles de ingresos no se compadecen con la extensión de la jornada de trabajo más allá de las 48 horas a la semana. El 40,1 \% de los TC de 2004 y el 49,5 \% de los TC de 2007 manifiestan haber laborado bajo estas circunstancias. En cuanto a la contratación laboral, la situación del grupo 
de 2007 pareciera haber mejorado en relación con los de 2004. En efecto, mientras que en este último año la ausencia de contratación ascendía al 27,6 \%, en 2007 dicha proporción fue del 20,7 \%; para aquellos que reportan algún tipo de contratación, la de carácter temporal representó en 2004 el 54,0 \% y en 2007 el 49,0 \%.

La dinámica del ciclo económico en cada actividad productiva, los cambios en la normatividad del trabajo y la reestructuración empresarial, inducen fuertes ritmos de rotación laboral que se manifiestan en la duración del ciclo empleo-desempleo de los TC. En efecto, mientras que en 2004 los TC reportan un promedio de 24,3 meses de permanencia en el último empleo, para el 2007 la misma desciende a un promedio de 18,1 meses. Entre tanto, el reporte de la duración del desempleo se muestra sensiblemente inferior en el primer año respecto de lo observado en 2007. Para el 45,5 \% de este último grupo de trabajadores la búsqueda de empleo se extiende más allá de un año, lo que significa que en el período más reciente estamos en presencia de una fuerte incidencia del desempleo de larga duración que habrá de incidir fuertemente en las posibilidades de mantener la afiliación al SGSSS.

Aseguramiento en salud según perfil sociolaboral de los TC de la ciudad de Medellín, 2004 y 2007

En cuanto al aseguramiento en salud, en 2004 el 38,2 \% de los TC reportó no estar afiliado, frente al 43,9 \% en 2007. Del 61,8 \% que estaba asegurado en 2004, dos tercios lo estaban en el régimen contributivo y el tercio restante en el subsidiado. Para 2007 estas proporciones fueron del 43,2 \% y el 56,8 $\%$ respectivamente. Entre los que se encontraban afiliados al régimen contributivo en 2004, el 79 \% lo estaba en condición de cotizante y el 21 \% de beneficiario, frente al 35,4 \% y 64,6 respectivamente en 2007.

En 2004, el 89,7 \% de los TC no afiliados al SGSSS manifestó estar dispuesto a acceder a recursos de un microcrédito para mantener su aseguramiento como cotizantes en el régimen contributivo en salud. Para conocer esta disposición, en aquel estudio se indagó a los TC sobre su disposición para acceder o no al "Subprograma de subsidio y de crédito reembolsable para garantizar el aseguramiento en salud y ayudar a proteger el patrimonio de los TC y de sus familias en el municipio de Medellín”, el cual fue presentado como alternativa de política pública ante el Concejo de esta ciudad, por parte del grupo de investigación. 
Tabla 2. Distribución porcentual de los TC según características socio-laborales y de aseguramiento en salud, Medellín 2004 y 2007

\begin{tabular}{|c|c|c|c|c|c|c|}
\hline \multirow[t]{2}{*}{ Variables } & \multicolumn{3}{|c|}{ Aseguramiento $2004 \%$} & \multicolumn{3}{|c|}{ Aseguramiento $2007 \%$} \\
\hline & Si & No & Valor P & $\mathrm{Si}$ & No & Valor P \\
\hline \multicolumn{7}{|l|}{ Sexo } \\
\hline Mujer & 64,4 & 35,6 & 0,063 & 63,6 & 36,4 & 0,004 \\
\hline Hombre & 45,1 & 54,9 & & 42 & 58 & \\
\hline \multicolumn{7}{|l|}{ Edad } \\
\hline 29 o menos años & 60,7 & 37,7 & 0,976 & 50 & 50 & 0,081 \\
\hline 30 a 39 años & 61,8 & 38,2 & & 56,8 & 43,2 & \\
\hline 40 o más años & 62,3 & 39,3 & & 69,6 & 30,4 & \\
\hline \multicolumn{7}{|l|}{ Educación } \\
\hline Primaria & 75 & 25 & 0,074 & 78,8 & 21,2 & 0,014 \\
\hline Secundaria & 59,7 & 40,3 & & 56,7 & 43,3 & \\
\hline Técnico-tecnológico & 59,4 & 40,6 & & 44 & 56 & \\
\hline Superior & 61,5 & 38,5 & & 48 & 52 & \\
\hline \multicolumn{7}{|c|}{ Estrato socioeconómico } \\
\hline Bajo & 66,7 & 33,3 & 0,033 & 61,7 & 38,3 & 0,084 \\
\hline Medio-alto & 53,5 & 46,5 & & 49,5 & 50,5 & \\
\hline \multicolumn{7}{|l|}{ Sector económico } \\
\hline Industria & 64,2 & 35,8 & 0,467 & 56,4 & 43,6 & 0,969 \\
\hline Comercio & 66,7 & 33,3 & & 55,7 & 44,3 & \\
\hline Resto & 58,5 & 41,5 & & 57,1 & 42,9 & \\
\hline \multicolumn{7}{|l|}{ Tamaño de empresa } \\
\hline 1 a 10 trabajadores & 64,7 & 35,3 & 0,504 & 58,7 & 41,3 & 0,605 \\
\hline $\begin{array}{l}\text { Más de } 10 \text { trabaja- } \\
\text { dores }\end{array}$ & 60,4 & 39,6 & & 54,8 & 45,2 & \\
\hline \multicolumn{7}{|l|}{ Posición ocupacional } \\
\hline Empleado s-privado & 50 & 50 & 0,225 & 53 & 47 & 0,076 \\
\hline Empleado s-público & 59,1 & 40,9 & & 64,3 & 35,7 & \\
\hline Resto (*) & 62,3 & 37,7 & & 81,3 & 18,8 & \\
\hline \multicolumn{7}{|l|}{ Salario último empleo } \\
\hline 1 smlv o menos & 64,6 & 35,4 & 0,184 & 66,2 & 33,8 & 0,016 \\
\hline Entre 1 y $1,5 \mathrm{smlv}$ & 63,3 & 36,7 & & 50,7 & 49,3 & \\
\hline Más de 1,5 smlv & 59,7 & 40,3 & & 49,1 & 50,8 & \\
\hline \multicolumn{7}{|c|}{ Duración del desempleo } \\
\hline Menos de 6 meses & 62,5 & 37,5 & 0,208 & 50,8 & 49,2 & 0,016 \\
\hline Entre 6 y 12 meses & 54,2 & 45,8 & & 42,6 & 57,4 & \\
\hline Más de 12 meses & 51,7 & 48,3 & & 66,7 & 33,3 & \\
\hline TOTAL & 61,8 & 38,2 & & 56,1 & 43,9 & \\
\hline
\end{tabular}

En 2007 se indagó a los TC su opinión sobre otra propuesta de política pública que sería presentada también a la Administración Municipal de Medellín denominada "Subprograma de subsidio de microseguro para garantizar la cotización y el aseguramiento en salud régimen contributivo 
como medida de protección social de los TC y de sus familias de los estratos sociales en condición de mediana pobreza en el municipio de Medellín”. De los no asegurados, el 82,3 \% manifestó su acuerdo con esta última propuesta la cual todavía se encuentra en proceso de revisión y discusión por parte del Concejo de la ciudad.

En la Tabla 2 se muestran las características de los TC según su afiliación o no al SGSSS. Conforme con los valores “p” de significación estadística al $5 \%$ y $10 \%$, gran parte de las diferencias de proporciones resultan relevantes, excepto las correspondientes a las variables sector económico, tamaño de empresa y edad de los TC. Esta última sólo tiene alguna relevancia para el año 2007.

Tabla 3. Distribución porcentual de los TC según características socio-laborales y de aseguramiento al régimen contributivo en salud, Medellín 2007

\begin{tabular}{|c|c|c|c|}
\hline Variable & $\begin{array}{l}\text { Aseguramiento } \\
\text { al régimen } \\
\text { contributivo } \\
n=48 \%\end{array}$ & $\begin{array}{l}\text { No aseguramiento } \\
\text { al régimen } \\
\text { contributivo } \\
n=150 \%\end{array}$ & Valor $\mathrm{P}$ \\
\hline \multicolumn{4}{|l|}{ Sexo } \\
\hline Mujer & $37(28,7)$ & $92(71,3)$ & 0,046 \\
\hline Hombre & $11(15,9)$ & $58(84,1)$ & \\
\hline \multicolumn{4}{|l|}{ Edad } \\
\hline 29 o menos años & $21(19,4)$ & $87(80,6)$ & 0,065 \\
\hline 30 a 39 años & $10(22,7)$ & $34(77,3)$ & \\
\hline 40 o más años & $17(37,0)$ & $29(63,0)$ & \\
\hline \multicolumn{4}{|l|}{ Educación } \\
\hline Primaria & $9(26,5)$ & $25(73,5)$ & 0,058 \\
\hline Secundaria & $24(25,3)$ & $71(74,7)$ & \\
\hline Técnico-tecnológico & $5(11,4)$ & $39(88,6)$ & \\
\hline Superior & $10(40,0)$ & $15(60,0)$ & \\
\hline \multicolumn{4}{|l|}{$\begin{array}{l}\text { Estrato socioeconó- } \\
\text { mico }\end{array}$} \\
\hline Bajo & $19(17,8)$ & $88(82,2)$ & 0,021 \\
\hline Medio-alto & $29(31,9)$ & $62(68,1)$ & \\
\hline \multicolumn{4}{|l|}{ Posición ocupacional } \\
\hline Empleado s-privado & $35(20,8)$ & $133(79,2)$ & 0,02 \\
\hline Empleado s-público & $5(35,7)$ & $9(64,3)$ & \\
\hline Resto $\left(^{*}\right)$ & $8(50,0)$ & $8(50,0)$ & \\
\hline
\end{tabular}




\section{DISCUSIÓN}

Los anteriores datos se complementan con la información de la tabla 3 que muestra las proporciones y sus diferencias estadísticamente significativas del aseguramiento o no aseguramiento de los TC en el régimen contributivo en 2007, las cuales presentaron alta relevancia para las variables sexo, estrato socioeconómico y posición ocupacional al momento de perder el empleo.

El perfil sociodemográfico de los TC de la ciudad de Medellín es similar para los dos periodos considerados en este estudio. La pérdida de empleo tiende a presentarse principalmente en jóvenes con bajos niveles de educación. Estos resultados son bastante coherentes con las tendencias históricas del desempleo a nivel nacional y local, pues está ampliamente documentada la fuerte vulnerabilidad al desempleo por parte de los jóvenes $(27,28)$. Por estrato socioeconómico, los perfiles observados en 2004 y 2007, en el sentido de una mayor incidencia del desempleo en la población de más bajos recursos, responden también a las características estructurales que han prevalecido en el mercado de trabajo del nivel nacional y de la ciudad de Medellín.

Por sexo y educación, se presentan algunas diferencias. En 2007 las mujeres se ven más afectadas por la pérdida del empleo que en 2004. Por niveles de educación, en 2007 se ve una alta participación de cesantes con formación técnica, tecnológica y superior que contrasta con la de 2004. Este último dato se distancia del comportamiento descendente en la tasa de desempleo de este grupo de trabajadores con alta formación a nivel nacional (5). Sin embargo, dicha circunstancia pudiera estar relacionada con la alta rotación laboral asociada a los procesos de incorporación de nuevas tecnologías que se acentúan con la expansión de la inversión en los sectores de la industria y los servicios modernos en la ciudad (29).

Las variables económicas y laborales también presentan importantes contrastes. Tanto en 2007 como en 2004, se encontró gran proporción de TC que en su último empleo laboraron como asalariados del sector público o del sector privado. La distribución por actividad económica presenta perfiles similares en ambos años, mostrando que es en los sectores de la industria y el comercio en donde se presenta una mayor rotación laboral, probablemente asociada también al cambio tecnológico, especialmente en 
la industria, lo cual se corresponde con la fuerte expulsión de trabajadores del sector privado moderno, más que del sector público o las microempresas.

En ambos periodos buena parte de los TC devengaban un SML o menos. Este dato refleja no sólo la precariedad en los ingresos sino también el hecho de que a un sector importante de trabajadores se les está violando su derecho en materia salarial. La vulneración de los derechos laborales es más evidente en relación con las formas contractuales, pues cerca del $50 \%$ estuvo trabajando bajo contratación temporal y no menos del $20 \%$ laboraba sin haber firmado explícitamente su relación contractual. Esto último revela las prácticas del empresariado nacional y local de forzar la contratación por fuera del código laboral, echando mano de la vinculación mediada por las cooperativas de trabajo asociado y/o la regulación del código civil y comercial (30).

Dicha situación se evidencia en otros estudios que señalan que cerca del $50 \%$ de los asalariados a nivel nacional no consigue formalizar su contrato laboral por escrito (31). Para el año 2000 esta proporción era cercana al 57 \% (32), lo cual indica una de las condiciones más aberrantes de precariedad del empleo en Colombia.

En el nivel agregado se evidencia una ampliación de la duración media del desempleo y una sensible reducción del tiempo de permanencia en el último empleo, lo cual contrasta con el hecho de que en 2007 la ciudad gozó de una mayor dinámica productiva y menores tasas de desempleo que en 2004. Se redujo el nivel de desempleo pero se amplió el período de búsqueda. Dicha circunstancia pudiera estar relacionada con el hecho de que dicha expansión económica no se reflejó en un aumento sustancial de las oportunidades de empleo, de modo que la reducción de la tasa de desempleo habría sido reflejo más bien de la reducción en la participación laboral en la ciudad (5).

Por lo demás, es igualmente probable que la dinámica laboral de la ciudad estuviera cambiando los incentivos de búsqueda y pronto vínculo laboral, en el sentido de que la expansión económica del último año pudo haber generado mayores oportunidades para los miembros principales del hogar, al punto que los miembros secundarios, sin urgencias para el enganche, se estuviesen tomando más tiempo en procura de las mejores ofertas de trabajo. 
En cuanto al aseguramiento en el SGSSS, cabe resaltar las altas proporciones de hombres, comparadas con las de las mujeres, que no logran mantener su afiliación a salud. Tanto en 2004 como en 2007, la desprotección en salud crece a medida que se desciende en los niveles de edad. El gradiente de desprotección es un tanto distinto con respecto a los niveles de educación, en tanto que se acentúa en los grados intermedios y superiores.

En el estrato medio-alto la desprotección en salud es mayor a la reportada en el nivel socioeconómico bajo. Un comportamiento similar se observa a medida que se asciende en los niveles salariales, sobre todo en lo correspondiente a lo registrado en 2007. Es bastante llamativa la dinámica de la protección social en salud en relación con la duración media del desempleo. El gradiente parece mostrar mayores proporciones de desprotección a medida que se extiende el período de cesantía.

Esta desprotección en salud presenta un incremento importante de los TC no asegurados: 43,9 \% en 2007 frente al 38,2 \% de 2004. Entre los TC que reportaron afiliación al SGSSS, conviene resaltar su distribución por régimen. Mientras en 2004 el 61,8 \% de los TC estaban asegurados, en 2007 sólo el 56,1 \% contaban con alguna afiliación. De otro lado, en 2007 el régimen subsidiado registró mayor proporción (56,8 \%) respecto al año 2004 (21,5 \%). Esto lo hemos considerado como una presión de los TC sobre los recursos financieros del régimen subsidiado dispuestos en el SGSSS para la población más pobre y vulnerable.

En 2004 el 21 \% de los afiliados al RC eran beneficiarios frente al $79 \%$ que estaban como cotizantes. Esto contrasta con los resultados de 2007 donde 64,6 \% eran beneficiarios frente al 35,4 \% como cotizantes, lo cual indica una pérdida de capacidad de pago de la cotización al Sistema por parte de esta población, lo que a su vez se corresponde con la mayor duración del periodo de cesantía en 2007.

En tal sentido, y dada la carencia de ingresos durante el tiempo de cesantía laboral, parece comprensible que el régimen subsidiado se convierta en la opción más posible de aseguramiento en salud para los TC que por la pérdida del empleo pasan de ser población cotizante del régimen contributivo a población pobre y vulnerable del subsidiado. 
Por lo anterior, consideramos que la vulnerabilidad de los TC y de su grupo familiar, demanda con prioridad la exploración y puesta en marca de alternativas de política pública para la protección en salud de esta población, diferentes al régimen subsidiado, que intervengan positivamente la desproporción existente entre los regímenes contributivo y subsidiado a causa de la precariedad laboral y la pérdida del empleo.

Limitaciones del Estudio:

Los resultados de los dos estudios aquí presentados sólo pueden extrapolarse a la población de trabajadores cesantes registrada en las bases de datos del SENA y en las cuatro EPS de la ciudad de Medellín que aportaron la información solicitada

Agradecimientos: A los trabajadores cesantes que participaron en ambos estudios, al IDRC (International Development Research Centre-Canadá), a la Secretaría de Salud de Medellín, a la Facultad Nacional de Salud Pública de la Universidad de Antioquia, a los Gerentes en Sistemas de Información en Salud, Jairo Martínez y Edwin Álvarez.

\section{REFERENCIAS}

1. Uthoff A. La protección Social de Cara al Futuro: Acceso, financiamiento y solidaridad. Montevideo: CEPAL; 2006. p 15.

2. Almeida C. Reforma de sistemas de servicios de salud y equidad en América Latina y el Caribe: algunas lecciones de los años 80 y 90. Cadernos de Saúde Pública. 2002; 18(4): 913.

3. Organización Panamericana de la Salud-Organización Mundial de la Salud. $26^{\mathrm{a}}$ Conferencia Sanitaria Panamericana. 54ª Sesión Del Comité Regional. Ampliación de la Protección Social en Materia de Salud: Iniciativa Conjunta de la Organización Panamericana de la Salud y la Organización Internacional del Trabajo. Washington, D.C.: OPS-OMS; 2002. p 4,7.

4. Madies C, Chiarvetti S, Chorny M. Aseguramiento y Cobertura: dos temas críticos en las reformas del sector de la salud. Revista Panamericana de Salud Pública 2000; 8(1/2): p 35.

5. República de Colombia. Departamento Administrativo Nacional de Estadística DANE. Encuesta Continua de Hogares, 2001-2007. Disponible en: http://www.dane.gov. co/daneweb_V09/index.php?option=com_content\&view=article\&id=121\&ltem id=67 Consultado febrero de 2009.

6. Medellín Como Vamos. Informe Pobreza y Desigualdad 2009. Disponible en: http://www. medellincomovamos.org/pobreza-y-desigualdad. Consultado mayo de 2010.

7. Nuñez J, Espinosa S. Determinantes de la pobreza y la vulnerabilidad. Estudio realizado para la Misión para el Diseño de una Estrategia para la Reducción de la Pobreza y la Desigualdad MERPD. 2005. Departamento Nacional de Planeación. Disponible en: http://www.dnp.gov.co/archivos/documentos/MP_En_Que_Vamos/Dets_pobreza_y_ 
vulnerabilidad_DOC.pdf Consultado septiembre de 2009.

8. Baltazar E, Álvarez S, Aguilar A. Riesgos Sociales, Movilidad Social y Trampas de la Pobreza de las Familias Pobres de Bogotá. En: Gutiérrez-Bonilla ML (Ed.). Las Familias en Bogotá. Realidades y Diversidades. Bogotá: Pontificia Universidad Javeriana; 2008. p 92.

9. Sojo A. Vulnerabilidad Social y Políticas Públicas. CEPAL Serie Estudios y PerspectivasSede Subregional de la CEPAL en México, 2004; 14: $p$ 9.

10. Restrepo J. El seguro de salud en Colombia. ¿Cobertura Universal? Revista Gerencia y Políticas de Salud. 2002; 2: p 29-31.

11. Martínez F, Robayo G, Valencia O. ¿Por qué no se logra la cobertura universal de la seguridad social en salud? Bogotá: FEDESALUD; 2002. p 129-131.

12. Nuñez J, Espinosa S. No siempre pobres, no siempre ricos: Vulnerabilidad en Colombia Documento CEDE 2005-15. ISSN 1657-719. Disponible en: http://economia. uniandes.edu.co/investigaciones y publicaciones/CEDE/Publicaciones/ documentos_cede/2005/no_siempre_pobres_no_siempre_ricos_vulnerabilidad_ en_colombia Consultado marzo de 2009.

13. Zambrano A, Ramírez M, Yepes F, Guerra J, Rivera D. ¿Qué muestran las Encuestas de Calidad de Vida sobre el sistema de salud en Colombia? Cadernos de Saúde Pública 2008; 24(1): p 124.

14. Organización Internacional del Trabajo. Empleo, un desafío para Colombia. Bogotá: OIT; 1999.

15. López H. Ensayos sobre economía laboral colombiana. Bogotá: Carlos Valencia editores y Fonade; 1996.

16. Lora E. El desempleo: ¿qué hacer? Alfaomega y cambio. Bogotá; 2001.

17. Farné S. Efectos de una reforma laboral en Colombia. Bogotá: Banco Interamericano de Desarrollo; 2002. (Mimeografiado)

18. Restrepo J. Empleo y seguridad social en Colombia: la necesidad de replantear una relación que se diluye. Medellín: Centro de Investigaciones Económicas, Universidad de Antioquia; 2001.

19. Nieto E. Factores socioeconómicos y aseguramiento en salud en el área urbana de Colombia. Revista Facultad Nacional de Salud Pública. 2001; 19(1): p 25-40.

20. Cardona A, Nieto E, Arbeláez MP et al. Impacto de la reforma de la seguridad social sobre la organización institucional y la prestación de los servicios de salud en Colombia. Medellín: FNSP, Universidad de Antioquia; 1997.

21. Rodríguez O. Reforma laboral y seguridad social en Colombia. Bogotá: Universidad Nacional; 1999

22. Organización de Naciones Unidas CEPAL. La protección Social de Cara al Futuro: Acceso, financiamiento y solidaridad. Montevideo: CEPAL; 2006. p 61.

23. Hernández M. Reforma Sanitaria, Equidad y Derecho a la Salud en Colombia. Cadernos Saúde Pública. 2002; 18(4): p. 998.

24. Franco A. Seguridad Social y Salud en Colombia. Estado de la Reforma. Revista de Salud Pública. 2000; 2(1): 1-16.

25. Barón G. Cuentas Nacionales de Salud en Colombia 1993-2003. El gasto nacional en salud y su financiamiento. Ministerio de la Protección Social. Programa de Apoyo a la Reforma de Salud PARS. Departamento Nacional de Planeación. Bogotá; 2007. p 92.

26. República De Colombia. Departamento Administrativo Nacional de Estadística DANE. Metodología de la Encuesta Nacional de Hogares. Conceptos utilizados en la ENH. Disponible en: http://www.dane.gov.co/index.php?option=com_content\&task=cate gory\&sectionid=19\&id=76\&ltemid=258 Consultado febrero de 2009.

27. Nieto E. Morfología del mercado laboral en Colombia. Revista Facultad Nacional de Salud Pública. 1998; 15(2): 9-43. 
28. Escuela Nacional Sindical. Informe Nacional de trabajo decente. Medellín: ENS; 2009. p 57-59.

29. Moreno A, Junca G, Bonilla $R$ et al. Bien-estar y macroeconomía: más allá de la retórica. Bogotá: Universidad Nacional de Colombia. Facultad de Ciencias Económicas; 2007. p 13-18.

30. Escuela Nacional Sindical. Informe Nacional de trabajo decente. Medellín: ENS; 2009. p 19.

31. Farné S, Granados E, Vergara CA. El mercado laboral y la seguridad social en Colombia entre finales del siglo XX y principios del siglo XXI. Bogotá, CEPAL. Serie Estudios y Perspectivas (15); 2006. Disponible en: http://www.eclac.org/publicaciones/ xml/3/27243/LCL.2618-P.pdf Consultado: agosto de 2009.

32. Uribe JI, Ortiz CH, García GA. Segmentación del mercado laboral colombiano en la década de los noventa. Archivos de Economía Departamento Nacional de Planeación República de Colombia. Documento 301; 2006. p. 24. 